BRAVILLIAN JOURNAL

www.bjournal.com.br
ISSN 0100-879X

Volume 43 (5) 381-496 May 2011

BIOMEDICAL SCIENCES

AND

CLINICAL INVESTIGATION

Braz J Med Biol Res, May 2011, Volume 44(5) 388-393

doi:

Cell therapy in dilated cardiomyopathy: from animal models to clinical trials

C. del Corsso and A.C. Campos de Carvalho

The Brazilian Journal of Medical and Biological Research is partially financed by

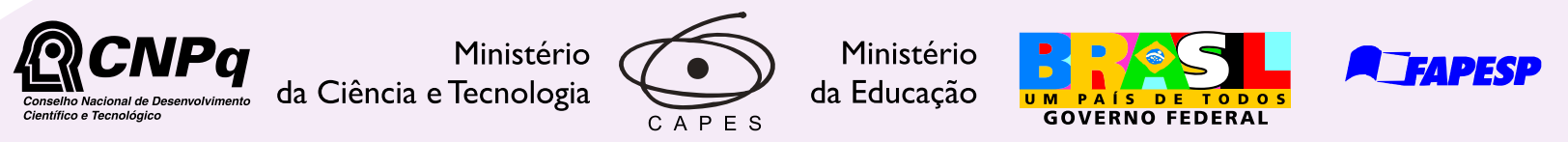

Institutional Sponsors
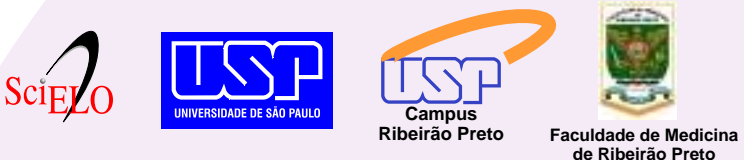

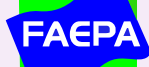

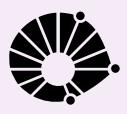

Ф SHIMADZU

GE Healthcare
Hotsite of proteomics metabolomics developped by:

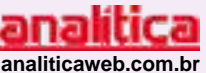

Thermo
SCIEN TIFIC 


\title{
Cell therapy in dilated cardiomyopathy: from animal models to clinical trials
}

\author{
C. del Corsso ${ }^{1}$ and A.C. Campos de Carvalho ${ }^{1,2}$ \\ ${ }^{1}$ Programa de Terapia Celular e Bioengenharia, Instituto de Biofísica Carlos Chagas Filho, \\ Universidade Federal do Rio de Janeiro, Rio de Janeiro, RJ, Brasil \\ ${ }^{2}$ Instituto Nacional de Cardiologia Larangeiras, Rio de Janeiro, RJ, Brasil
}

\begin{abstract}
Dilated cardiomyopathy can be the end-stage form and common denominator of several cardiac disorders of known cause, such as hypertensive, ischemic, diabetic, and Chagasic diseases. However, some individuals have clinical findings, such as an increase in ventricular chamber size and impaired contractility (classical manifestations of dilated cardiomyopathy) even in the absence of a diagnosed primary disease. In these patients, dilated cardiomyopathy is classified as idiopathic, since its etiology is obscure. Nevertheless, regardless of all of the advances in medical, pharmacological and surgical procedures, the fate of patients with dilated cardiomyopathy (of idiopathic or of any other known cause) is linked to arrhythmic episodes, severe congestive heart failure and an increased risk of sudden cardiac death. In this review, we will summarize present data on the use of cell therapies in animal models of dilated cardiomyopathies and will discuss the few clinical trials that have been published so far involving patients affected by this disease. The animal models discussed here include those in which the cardiomyopathy is produced by genetic manipulation and those in which disease is induced by chemical or infectious agents. The specific model used clearly creates restrictions to translation of the proposed cell therapy to clinical practice, insofar as most of the clinical trials performed to date with cell therapy have used autologous cells. Thus, translation of genetic models of dilated cardiomyopathy may have to wait until the use of allogeneic cells becomes more widespread in clinical trials of cell therapies for cardiac diseases.
\end{abstract}

Key words: Dilated cardiomyopathy; Stem cell; Heart failure; Chagas' disease; Cell therapy

\section{Introduction}

The term cardiomyopathy has been used for more than 5 decades to designate diseases that directly affect the cardiac muscle, inducing cardiomegaly and heart failure (1). Until 2006, the cardiomyopathies were divided into three major groups: dilated, hypertrophic and restrictive, according to anatomical and functional criteria (2). This division has been criticized for providing little information in view of the growing knowledge of the underlying causes of the different cardiomyopathies. Furthermore, at times a hypertrophic cardiomyopathy will lead to both a dilated and restrictive phenotype. Thus, the American Heart Association (3) and the European Society of Cardiology (4) have proposed new, although not consensual classifications for the cardiomyopathies. Relevant to this review is the fact that both classifications maintained the term dilated cardiomyopathy (DCM) attributable to genetic or non-genetic mechanisms.

Of even greater importance for the reader of this review is the understanding that the DCMs as defined here exclude those caused by ischemic diseases of the heart and/ or hypertension, being restricted to diseases that directly affect the myocardium. Even though DCMs of ischemic origin account for the majority of the cases of heart failure, the DCMs are responsible for about $30 \%$ of all cases of chronic heart failure in the patient population. Since heart failure has reached epidemic levels around the world and in the US alone its prevalence among adults aged 20 years or more is estimated at 5.3 million people, reaching about $12 \%$ of the population aged 80 years and older (5), the $30 \%$ figure for heart failure due to DCM represents a major health problem. Furthermore, conventional therapy for DCM (pharmacological and devices), although having had a major impact on life quality and survival, is able only to stop disease progression and in many cases fails, leaving as a last option for the patient heart transplantation. The shortage of donors, the problems of immune suppression

Correspondence: C. del Corsso, Instituto de Biofísica Carlos Chagas Filho, UFRJ, Av. Carlos Chagas Filho, 373, CCS, BI. G2-045, 21941-902 Rio de Janeiro, RJ, Brasil. Fax: +55-21-2280-8193. E-mail: cdcorsso@biof.ufrj.br (Cc: cdcorsso@gmail.com)

Received January 28, 2011. Accepted March 24, 2011. Available online April 15, 2011. Published May 16, 2011. 
and the high cost of the procedure have turned cardiac transplantation into a limited therapeutic option. On this scenario, new therapies are needed and the use of stem cells to regenerate the diseased heart is being intensively investigated.

Here we will discuss data on the use of cell therapies in animal models of DCM and the few clinical trials that have been published so far involving patients affected by this disease.

\section{Cell therapy in animal models of dilated cardiomyopathy}

The idea of using cell transplantation as an alternative therapy to restore myocardial function started in the early 90's. At that time, the prevalent idea was that the heart was a post-mitotic organ and, therefore, unable to regenerate. Based on that argument, independent groups started to treat infarcted heart tissue with different cell types (mostly already fully differentiated into cardiomyocytes) in order to recover systolic and/or diastolic function. In fact, the overall conclusion from those initial experiments was that the different transplanted cell types induced an improvement in heart function compared with findings in control hearts (6-10). The majority of reports, however, did not attribute the observed functional effects to the grafted cells themselves, since cell types such as HL-1 cells, fetal and neonatal cardiomyocytes, did not engraft into the scarred heart tissue (10).

Soon after the first positive results in ischemic animal models were published, many investigators attempted to show that cell transplantation could also benefit failing hearts due to DCM. Using distinct models of DCM, Scorsin et al. (11) and Yoo et al. (12) have shown that direct intramyocardial injection of fully differentiated cardiomyocytes was able to form cardiac-like tissue and improve the contractile function of globally failing hearts. In the first study, a recovery in heart function was produced in a mouse model of doxorubicininduced heart failure treated with fetal and adult mouse cardiomyocytes. Yoo et al. (12) used a genetically modified hamster model (BIO 53.58) in which animals were injected with 4 million adult hamster myocytes in the left ventricular anterior free wall. Four weeks after transplantation, the lack and/or low number of transplanted cardiomyocytes into host tissue led the authors to suggest that the observed effects could not be directly related to engraftment of the injected cells, but instead to the cardioprotective factors released by them.

Using multiple injection sites rather than a single intramyocardial injection site, Pouly et al. (13) demonstrated a significant improvement in left systolic function in CHF147 hamsters 4 weeks after treatment with skeletal myoblasts. One of the major concerns about this approach was that, although multiple sites of injections could help by spreading the transplanted cells throughout the failing heart, the existing fibrosis could be worsened by the inflammatory reaction triggered by multiple needle punctures. However, morphological analysis carried out shortly after cell injection consistently showed skeletal myoblasts engrafted into the transplanted hearts without signs of additional fibrosis. In order to obtain a better dissemination of transplanted cells into dilated hearts, since DCM is a widespread, non-focal disease of the heart, Suzuki et al. (14) injected one million syngeneic (but not autologous) myoblasts into the coronary arteries of doxorubicin-induced dilated and failing rat hearts. One month later, Langendorff experiments revealed a $17 \%$ improvement of both systolic and diastolic function measured by positive and negative $\mathrm{dP} / \mathrm{dt}$, respectively, when compared to non-injected hearts. In addition, and in contrast to previous findings, immunohistochemistry analysis of injected hearts showed a widespread engraftment of myoblasts and differentiation into multinucleated myotubes axially aligned with host cardiac fibers. More recently, Hata et al. (15) developed and tested tissue-engineered myoblast patches in a canine model of DCM. After 4 weeks of pacing-induced heart failure, 5 dogs received about 20 patches containing 1.5-2.5 million cells per patch. When compared to sham-operated animals $(\mathrm{N}=7)$, transplanted dogs showed a marked improvement in left ventricular ejection fraction (EF) and fractional shortening (33.3 and 229\%, respectively) with reduced ventricular chamber volume and a $27 \%$ increase in wall thickening.

In 2005, Werner et al. (16) used an autoimmune model of myocarditis, in which rats immunized against myosin developed DCM 6 weeks after a booster injection. By assessing the number and adhesion properties of endothelial progenitor cells (EPCs), the authors concluded that, despite the higher number of colony forming units from the spleen pool of DCM rats, both spleen- and bone marrow-derived EPCs from DCM rats were functionally compromised compared to healthy rats. According to the authors, the dysfunctional state of EPCs could contribute to the already known myocardial damage and malfunction characteristic of this autoimmune model of DCM. This was further confirmed when a considerable improvement in myocardial performance (a $15 \%$ increase in fractional shortening) was detected in DCM rats that received EPCs. This gain of function was attributed to the correction of the quantitative and qualitative defects of EPCs.

Roura et al. (17) recently showed that patients with DCM also have higher numbers of circulating EPCs and $\mathrm{CD}_{133^{+}} /$ VEGF-R2 ${ }^{+}$cells (a subset of EPCs that can differentiate into mature endothelial cells) compared to normal individuals. The investigators did not evaluate the functional properties of these cells, but concluded that there is a defective vascularization and impaired vasculogenesis and angiogenesis in humans with DCM. This is in agreement with Werner et al. (16) who reported the same results although not observing differences in vascular density between EPC-treated and -untreated rats, even though the intravenous injection of 
EPCs in DCM rats resulted in a $15 \%$ increase in contractility and attenuation of scar formation (16). The potential benefit induced by EPC transplantation had already been described for myocardial (18) and hind limb (19) ischemic models, but the single or multiple mechanisms by which those beneficial effects were achieved is still an unanswered question.

Using the same animal model, but a different type, number and cell delivery method, Nagaya et al. (20) showed that 5 million mesenchymal stem cells (MSC) directly injected into 10 different points of a diseased myocardium resulted in a $24 \%$ decrease in left ventricular end-diastolic pressure and in an improvement of around $45 \%$ in heart contractility (increased maximum dP/dt) 4 weeks after treatment. The investigators attributed the observed effects to both the ability of MSC to differentiate into cardiomyocytes, vascular endothelial and smooth muscle cells and to their capacity to secrete large amounts of angiogenic, antiapoptotic and mitogenic factors that exert a cardioprotective effect.

Using the TO-2 hamster strain, a representative model of a myopathic animal that mimics the hereditary human DCM, Kondoh et al. published a series of three papers (2123) that demonstrates how far we are from finding the "ideal treatment" for DCM. In all of them, male 27-week-old TO-2 hamsters showing moderate cardiac remodeling were used as recipients. In the first study (21), the authors showed that TO-2 hamsters treated with liposomes containing human hepatocyte growth factor (HGF) cDNA had an improvement of left ventricular end-systolic dimension, fractional shortening and a decrease of almost $47 \%$ in myocardial fibrosis. Moreover, an increase in survival rate associated with transient reorganization of the cytoskeletal proteins was also observed, leading the authors to propose the HGF as an alternative treatment for DCM. One year later, the same group tested the effects of a sheet-shaped myoblast graft versus a direct injection of those cells on cardiac performance of TO-2 hamsters (22). In this case, myoblasts were harvested from the lower limbs of BIO F1B hamsters and a total of 10 million cells were injected directly with a 30-G tuberculin syringe or delivered as a sheet-shaped graft. The authors highlighted a reduction in left ventricular dimension for the animals treated with the sheet-shaped graft, whereas a progression of left ventricular dilation was observed in the cell-treated hamsters. Likewise, fractional shortening worsened in both the sham-operated and celltreated groups, while this parameter was kept constant at the preoperative level for three months after the procedure in the sheet-shaped-treated animals. Interestingly, an increase in life expectancy and reorganization of the cytoskeletal proteins were also observed only in those animals. In 2007 (23), human HGF gene transfection was applied alone or combined with myoblasts (harvested from the lower limbs of BIO F1B hamsters) to diseased (TO-2) animals. At that time, a total of 20 million myoblasts were evenly injected into four different heart sites (in the right ventricle and at the apex and on the anterior and posterior walls of the left ventricle). Cardiac performance was evaluated biweekly until the animals' death (when the histological data were obtained). Both myoblast transplantation and HGF gene transfection improved the cardiac function of DCM hamsters, but the association of both interventions preserved the systolic function better than either treatment alone. Again, the lifespan of TO-2 hamsters was significantly prolonged in both the groups treated with HGF alone and with HGF plus myoblast transplantation when compared to vehicleand myoblast-injected groups. The authors suggested that the combination therapy (HGF + myoblast transplantation) is a promising treatment for human DCM but they never mentioned the arrhythmogenic potential of a myoblast graft inside the heart, since this graft lacks connexin 43 expression that is essential for intercellular communication and electrical propagation in the heart.

More recently a mouse model of doxorubicin-induced DCM was used to evaluate a new cardiac stem/progenitor cell candidate to ameliorate a dysfunctional heart (24). In this model, embryonic GFP ${ }^{+}$stem cells (ES) from a genetically modified C57/B6 mouse (Gact4) were cultured in a differentiation medium for 4 days and selected according to expression of Flk1 protein, a marker of early mesodermal cells that is reported to be associated with differentiation into cardiomyocytes. Indeed, when Gact4-derived Flk1+ cells were injected directly into 20 DCM mouse hearts, functional analysis revealed a significant improvement of both systolic and diastolic function of DCM mice compared to sham and control groups. Moreover, immunohistochemical assays showed that Flk1+ cells were found engrafted within ventricular cardiomyocytes and, from an electrophysiological point of view, those cells behaved exactly like adult ventricular cells, rather than as embryonic or neonatal cardiomyocytes. However, it is well known that a major concern when dealing with ES is the generation of tumors. In this protocol, the authors transplanted those cells in an extra group of 5 mice and followed them for 10 weeks. At the end, the animals were sacrificed and no signs of abnormal growths or proliferation activity (detected by the presence of Ki-67) were detected, supporting the authors' hypothesis that, at that point, ES cells had already fully differentiated into adult cardiomyocytes.

Gene therapy has also been used to protect against or prevent the myocardial injury observed in cardiomyopathic animal models. Since myocardial fibrosis and changes in either cytoskeletal or sarcolemmal proteins are responsible for the global remodeling often found in patients and animal models with DCM, the expression and/or deletion of specific proteins could play an important role in the functional recovery of failing hearts. In this scenario, HGF, a mesenchymal-derived factor that regulates cell growth, motility and morphogenesis and that is also known to prevent fibrosis in liver and pulmonary injury models, was used to inhibit the progression of cardiac fibrosis in a cardiomyopathic hamster lineage (BIO 14.6). The human HGF gene was 
injected directly into the myocardium of BIO 14.6 hamsters and the animals were evaluated weekly from week 12 to 20 (25). In that study, Taniyama et al. (25) showed that HGF transfection was able to significantly decrease the fibrotic area by $27 \%$ and increase the capillary density by $33 \%$ and the blood flow in the heart. However, in contrast to a report by Kondoh et al. (21), it was not possible to evaluate the functional effects of HGF overexpression at that time since BIO 14.6 hamsters do not show myocardial dysfunction at the age of 20 weeks.

In summary, a wide variety of cell types have been used in animal models of DCM: embryo-derived stem cells, fully differentiated cardiomyocytes, skeletal myoblasts, EPC, MSC and so on. The use of the first two cell types in clinical trials is still problematic, due to the possibility of tumor formation for the former and due to immune and availability restrictions for the latter. Autologous skeletal myoblasts have been used in clinical trials, but development of fatal arrhythmias in some patients has put these trials on hold. EPCs and MSC have also been used in humans with ischemic heart disease and the results of phase I/II trials have been encouraging even though efficacy has not been proved. Therefore, the scenario emerging from the animal studies has led a few groups to attempt the use of bone marrowderived cells in patients with DCM. These trials have been largely concerned with the safety of the procedure and are summarized in the next section.

\section{Cell therapy in patients with dilated cardiomyopathy}

Although a sizable number of trials, involving close to 1000 patients, including some randomized and blind trials, have already been performed in the setting of ischemic cardiopathies, very few cell therapy trials have been performed in patients with non-ischemic dilated cardiomyopathy. All reported DCM trials are Phase I trials, addressing the question of safety and feasibility. In 2006, Arguero et al. (26) reported a trial in which 39 terminal heart failure patients were subjected to cell therapy by direct injection of circulating cells collected by hemopheresis after granulocyte colony-stimulating factor mobilization into the myocardium by mini-thoracotomy. However, only 5 of the 39 patients had non-ischemic DCM in the group. Given the high number of patients developing ventricular fibrillation (7) and 2 deaths during the perioperative period, the safety of the procedure is questionable. Using the same injection route Kalil et al. (27) registered no adverse events in 9 patients with idiopathic DCM treated with the mononuclear fraction of bone marrow. Six patients who completed a 1-year follow-up experienced a transient improvement in heart function, measured by EF in echocardiography 2 months after cell injection, but MRI failed to show variation in EF\%. At 6 and 12 months after cell injection this gain in EF\% had vanished even when determined by echocardiography, although quality of life and functional class were still reported to be significantly improved by the authors. Using the intracoronary route with balloon inflation as described by Strauer et al. (28), Widimsky and Penicka (29) reported serious adverse events during the procedure, leading to interruption of the trial after treating the second DCM patient. In both cases, patients could not withstand the balloon inflation maneuver, which led to severe arrhythmias in one case and severe hypotension with coronary spasm in the other. Still in 2006, Seth et al. (30) reported a randomized trial involving 44 patients with dilated cardiomyopathy of non-ischemic origin. Twenty-four patients were assigned to cell therapy and the remaining 20 patients to the control group. Patients were followed up for 12 months, although the results reported refer only to the first 6 months of follow-up. Cell therapy used the mononuclear fraction of bone marrow. Cells were injected into the coronary circulation after inflating a balloon in the coronary sinus to slow coronary flow. The total number of cells injected per patient or the mean number of injected cells was not reported. However, cells were viable (99\% viability) and the percentage of $\mathrm{CD}_{3}{ }^{+}$was rather high in the mononuclear fraction (close to 5\%). Patients were monitored by holter, echocardiogram and biopsies at baseline and during followup. Mortality was similar in both the cell-treated and control groups, but a $5.4 \%$ improvement in the absolute value of the EF (by echocardiogram using Simpson's rule) was reported for the cell group while the control group showed no variation in EF\%. Interestingly, looking at individual patients (as shown in Figure 1 of the cited paper), 7 of the 20 surviving patients who were followed for 6 months either did not show improvement in EF\% or had a very small increase in EF\%. Two Chinese publications $(31,32)$ in the same year reported trial results using bone marrow mononuclear cells and MSC in patients with idiopathic dilated cardiomyopathy (IDC). A total of 42 patients ( 24 and 18 from Wang et al. and Huang et al., respectively) were enrolled in these trials. In the first study, 12 patients received MSC while in the second study mononuclear cells were injected in 10 patients. Both groups used the intracoronary route but the abstract did not state if balloon inflation was used or not (articles are in Chinese). No adverse events were reported in either case during the procedure and there was no increase in EF\% detected by echocardiography. However, a 6-min walking test showed a significant improvement of 31 and $11 \%$ in the cell group compared to baseline and to the control group, respectively.

Our own experience with cell therapy in IDC patients started in 2005 when we enrolled the first of 25 patients for a phase I trial with intracoronary injection of the bone marrow mononuclear fraction (33). Major exclusion criteria were coronary artery disease, chagasic cardiomyopathy, and any conditions that might affect survival such as renal or liver failure. To be included, patients had to be in classes II-IV of the New York Heart Association (NYHA), with EF\% below $35 \%$ by echocardiography, on optimized 
pharmacologic therapy and diagnosed as having IDC. After aspiration of 50-100 mL marrow from the iliac crest under sedation and local anesthesia, cells were processed in a Ficoll density gradient and the mononuclear fraction was isolated. Cell number and viability were determined and the cells were resuspended in $20 \mathrm{~mL}$ saline with $5 \%$ autologous serum. The cell suspension, in two $10-\mathrm{mL}$ syringes, was then sent to the catheterization laboratory for injection. The whole procedure from aspiration to cell injection lasted 2.5-3 h. Before cell injection, patients were subjected to cineangiocoronariography and 1 of the 25 patients was excluded due to the presence of CAD. In the remaining 24 patients subjected to cell injection, 3 adverse events were recorded. One patient developed increased enzyme levels (troponin and creatine kinase-MB) transiently without pain, ECG or hemodynamic instability, and 2 patients showed transient arrhythmias during coronary cell infusion, right bundle branch block in one and atrial fibrillation (reversed with amiodarone) in the other. During the first catheterization, endomyocardial biopsies were obtained in the right septal region and this procedure was repeated when patients were subjected to left ventricular angiography at the end of the study. Biopsies revealed little inflammatory infiltrate and variable amounts of fibrosis, which did not change with cell therapy. In 3 patients, injected cells were labeled with technetium and their localization was studied by scintigraphy, revealing a small amount of cell retention in the cardiac area within $24 \mathrm{~h}$ of cell injection. As was the case in the study of Seth et al. (30), 4 of our patients died during follow-up. All deaths occurred at least two months after the procedure and were unlikely to be related to cell

\section{References}

1. World Health Organization. Report of the WHO/ISFC task force on the definition and classification of cardiomyopathies. Br Heart J 1980; 44: 672-673.

2. Braunwald E. Cardiology: how did we get here, where are we today and where are we going? Can J Cardiol 2005; 21: 1015-1017.

3. Maron BJ, Towbin JA, Thiene G, Antzelevitch C, Corrado D, Arnett $\mathrm{D}$, et al. Contemporary definitions and classification of the cardiomyopathies: an American Heart Association Scientific Statement from the Council on Clinical Cardiology, Heart Failure and Transplantation Committee; Quality of Care and Outcomes Research and Functional Genomics and Translational Biology Interdisciplinary Working Groups; and Council on Epidemiology and Prevention. Circulation 2006; 113: 1807-1816.

4. Elliott $P$, Andersson B, Arbustini E, Bilinska Z, Cecchi F, Charron P, et al. Classification of the cardiomyopathies: a position statement from the European Society of Cardiology Working Group on Myocardial and Pericardial Diseases. Eur Heart J 2008; 29: 270-276.

5. Rosamond W, Flegal K, Furie K, Go A, Greenlund K, Haase $\mathrm{N}$, et al. Heart disease and stroke statistics - 2008 update: injection. In contrast to the study of Seth et al. (30), we did not observe improvement of mean EF by three different methods: echocardiography, MRI and left ventricular angiography. However, 5 of 19 patients on whom an MRI was performed (the gold standard) showed improvement in EF\% of $5 \%$ or more. In agreement with results reported by the Chinese groups $(31,32)$, who also did not observe an increase in EF\%, we found a significant increase of distance $(18 \%)$ in the 6 -min walk test as well as a significant increase in maximal oxygen consumption (29\%), test duration and test distance by ergospirometry. NYHA class, as well as the score for the Minnesota Quality of Life Questionnaire, also improved significantly (33).

The picture emerging from the few trials performed on patients to date can be summarized as follows: except for the occurrence of complications with intracoronary cell delivery using a balloon, the procedure seems safe; sustained arrhythmias have been reported in the trials (all used bone marrow-derived cells); trials were not designed to test for functional cardiac improvement, but the results suggested that larger randomized, double-blind and placebo-controlled trials should be performed to test for efficacy. In fact, two such trials are currently underway: one in Brazil and one in India. We expect that such trials will provide information about the efficacy of this therapy in DCM of non-ischemic origin.

\section{Acknowledgments}

Research supported by FAPERJ, CNPq, and Ministério da Saúde (Decit). a report from the American Heart Association Statistics Committee and Stroke Statistics Subcommittee. Circulation 2008; 117: e25-e146.

6. Chiu RC, Zibaitis A, Kao RL. Cellular cardiomyoplasty: myocardial regeneration with satellite cell implantation. Ann Thorac Surg 1995; 60: 12-18.

7. Li RK, Jia ZQ, Weisel RD, Mickle DA, Zhang J, Mohabeer MK, et al. Cardiomyocyte transplantation improves heart function. Ann Thorac Surg 1996; 62: 654-660.

8. Li RK, Jia ZQ, Weisel RD, Merante F, Mickle DA. Smooth muscle cell transplantation into myocardial scar tissue improves heart function. J Mol Cell Cardiol 1999; 31: 513522.

9. Taylor DA, Atkins BZ, Hungspreugs P, Jones TR, Reedy MC, Hutcheson KA, et al. Regenerating functional myocardium: improved performance after skeletal myoblast transplantation. Nat Med 1998; 4: 929-933.

10. Watanabe E, Smith DM Jr, Delcarpio JB, Sun J, Smart FW, Van Meter $\mathrm{CH}$ Jr, et al. Cardiomyocyte transplantation in a porcine myocardial infarction model. Cell Transplant 1998; 7: 239-246.

11. Scorsin M, Hagege AA, Dolizy I, Marotte F, Mirochnik N, 
Copin $\mathrm{H}$, et al. Can cellular transplantation improve function in doxorubicin-induced heart failure? Circulation 1998; 98 : II-151-II-155.

12. Yoo KJ, Li RK, Weisel RD, Mickle DA, Jia ZQ, Kim EJ, et al. Heart cell transplantation improves heart function in dilated cardiomyopathic hamsters. Circulation 2000; 102: III-204III-209.

13. Pouly J, Hagege AA, Vilquin JT, Bissery A, Rouche A, Bruneval $P$, et al. Does the functional efficacy of skeletal myoblast transplantation extend to nonischemic cardiomyopathy? Circulation 2004; 110: 1626-1631.

14. Suzuki K, Murtuza B, Suzuki N, Smolenski RT, Yacoub MH. Intracoronary infusion of skeletal myoblasts improves cardiac function in doxorubicin-induced heart failure. Circulation 2001; 104: I-213-I-217.

15. Hata H, Matsumiya G, Miyagawa $S$, Kondoh H, Kawaguchi N, Matsuura N, et al. Grafted skeletal myoblast sheets attenuate myocardial remodeling in pacing-induced canine heart failure model. J Thorac Cardiovasc Surg 2006; 132: 918-924.

16. Werner L, Deutsch V, Barshack I, Miller H, Keren G, George J. Transfer of endothelial progenitor cells improves myocardial performance in rats with dilated cardiomyopathy induced following experimental myocarditis. J Mol Cell Cardiol 2005; 39: 691-697.

17. Roura S, Planas F, Prat-Vidal C, Leta R, Soler-Botija C, Carreras $F$, et al. Idiopathic dilated cardiomyopathy exhibits defective vascularization and vessel formation. Eur $J$ Heart Fail 2007; 9: 995-1002.

18. Kawamoto A, Gwon HC, Iwaguro H, Yamaguchi JI, Uchida S, Masuda $\mathrm{H}$, et al. Therapeutic potential of ex vivo expanded endothelial progenitor cells for myocardial ischemia. Circulation 2001; 103: 634-637.

19. Takahashi T, Kalka C, Masuda H, Chen D, Silver M, Kearney $M$, et al. Ischemia- and cytokine-induced mobilization of bone marrow-derived endothelial progenitor cells for neovascularization. Nat Med 1999; 5: 434-438.

20. Nagaya N, Kangawa K, Itoh T, Iwase T, Murakami S, Miyahara $\mathrm{Y}$, et al. Transplantation of mesenchymal stem cells improves cardiac function in a rat model of dilated cardiomyopathy. Circulation 2005; 112: 1128-1135.

21. Kondoh H, Sawa Y, Fukushima N, Matsumiya G, Miyagawa S, Kitagawa-Sakakida S, et al. Reorganization of cytoskeletal proteins and prolonged life expectancy caused by hepatocyte growth factor in a hamster model of late-phase dilated cardiomyopathy. J Thorac Cardiovasc Surg 2005; 130: 295-302.

22. Kondoh H, Sawa $\mathrm{Y}$, Miyagawa S, Sakakida-Kitagawa S, Memon IA, Kawaguchi N, et al. Longer preservation of cardiac performance by sheet-shaped myoblast implantation in dilated cardiomyopathic hamsters. Cardiovasc Res 2006; 69: 466-475.
23. Kondoh H, Sawa Y, Fukushima N, Matsumiya G, Miyagawa S, Kitagawa-Sakakida S, et al. Combined strategy using myoblasts and hepatocyte growth factor in dilated cardiomyopathic hamsters. Ann Thorac Surg 2007; 84: 134-141.

24. Baba S, Heike T, Yoshimoto M, Umeda K, Doi H, Iwasa T, et al. Flk1(+) cardiac stem/progenitor cells derived from embryonic stem cells improve cardiac function in a dilated cardiomyopathy mouse model. Cardiovasc Res 2007; 76: 119-131.

25. Taniyama Y, Morishita R, Aoki M, Hiraoka K, Yamasaki K, Hashiya $\mathrm{N}$, et al. Angiogenesis and antifibrotic action by hepatocyte growth factor in cardiomyopathy. Hypertension 2002; 40: 47-53.

26. Arguero R, Careaga-Reyna G, Castano-Guerra R, GarridoGarduno MH, Magana-Serrano JA, de Jesus Nambo-Lucio. Cellular autotransplantation for ischemic and idiopathic dilated cardiomyopathy. Preliminary report. Arch Med Res 2006; 37: 1010-1014.

27. Kalil RA, Ott D, Sant'Anna R, Dias E, Marques-Pereira JP, Delgado-Canedo $A$, et al. Autologous transplantation of bone marrow mononuclear stem cells by mini-thoracotomy in dilated cardiomyopathy: technique and early results. São Paulo Med J 2008; 126: 75-81.

28. Strauer BE, Heidland UE, Heintzen MP, Schwartzkopff B. Pharmacologic myocardial protection during percutaneous transluminal coronary angioplasty by intracoronary application of dipyridamole: impact on hemodynamic function and left ventricular performance. J Am Coll Cardiol 1996; 28: 1119-1126.

29. Widimsky P, Penicka M. Complications after intracoronary stem cell transplantation in idiopathic dilated cardiomyopathy. Int J Cardiol 2006; 111: 178-179.

30. Seth S, Narang R, Bhargava B, Ray R, Mohanty S, Gulati G, et al. Percutaneous intracoronary cellular cardiomyoplasty for nonischemic cardiomyopathy: clinical and histopathological results: the first-in-man ABCD (Autologous Bone Marrow Cells in Dilated Cardiomyopathy) trial. J Am Coll Cardiol 2006; 48: 2350-2351.

31. Wang JA, Xie XJ, He H, Sun Y, Jiang J, Luo RH, et al. [A prospective, randomized, controlled trial of autologous mesenchymal stem cells transplantation for dilated cardiomyopathy]. Zhonghua Xin Xue Guan Bing Za Zhi 2006; 34: 107-110.

32. Huang RC, Yao K, Li YL, Zhang YQ, Xu SK, Shi HY, et al. [Transplantation of autologous bone marrow mononuclear cells on patients with idiopathic dilated cardiomyopathy: early results on effect and security]. Zhonghua Xin Xue Guan Bing Za Zhi 2006; 34: 111-113.

33. Martino HF, Oliveira PS, Souza FC, Costa PC, Assunção e Silva E, Villela R, et al. A safety and feasibility study of cell therapy in dilated cardiomyopathy. Braz J Med Biol Res 2010; 43: 989-995. 